\title{
The Optimal Sequence of Prices and Auctions
}

\author{
[Extended Abstract] ${ }^{*}$
}

\author{
Hanzhe Zhang \\ Department of Economics \\ University of Chicago \\ hanzhe@uchicago.edu
}

\begin{abstract}
Although in theory a second-price auction with a carefully chosen reserve price generates the optimal revenue when buyers have independent private values, in practice it could be costly to run such an auction. Compared to a simple posted price, an auction is involved with sellers' operational costs and buyers' mental costs of bidding.

This paper takes into account auction costs for both sellers and buyers, and investigates how a seller repeatedly chooses between prices and auctions to maximize her expected profit in a dynamic environment. Specifically, a seller must sell one unit of an indivisible good within $T$ periods and in each period, she either runs a reserve price auction incurring a per-period auction cost or posts a price for free. $T$ can be one, finite, infinite, or stochastic. Buyers with independent private values enter the market each period and pay a cost to bid in an auction. Buyers can be short-lived or long-lived, myopic or forward-looking.
\end{abstract}

Unsurprisingly, the seller in the static setting when all buyers arrive at once runs an auction with the Myerson optimal reserve price if the auction cost is low and posts a price if the auction cost is high; in the dynamic setting, a sequence of auctions with declining reserve prices is optimal when the auction cost is sufficiently low, and a sequence of declining prices is optimal when the auction cost is sufficiently high. When the auction cost is within the most economically relevant range, the optimal mechanism sequence takes a neat form: prices followed by auctions. No other complicated combination of prices and auctions though feasible is optimal; for example, it is never optimal to run auctions then post prices. The prices-then-auctions sequence resembles the buy-it-now selling mechanism on eBay: the seller posts a price at which any buyer can snatch the good before an auction starts and the price disappears.

${ }^{*}$ A full version of this paper is available at http://ssrn. com/abstract $=2401469$
The key result, the optimality of the prices-then-auctions sequence, relies on an endogenous opportunity cost unique to auctions in the dynamic setting. In the static setting, the seller only faces the tradeoff between an auction's revenue advantage and its additional operational cost. In the dynamic setting, the seller faces an additional cost when he uses an auction. The good not sold today is worth the expected revenue it generates in the next period. Since the probability of sale is higher using the optimal auction than using the optimal posted price, using an auction in a dynamic setting incurs not only an operational cost but also a higher opportunity cost associated with selling the good early. The operational cost stays constant but the opportunity cost decreases over time. As a result, running an auction in a later period incurs a lower opportunity cost than in an earlier period, and therefore is more desirable.

Having understood the auction's endogenous declining opportunity cost, we can see that the optimal prices-thenauctions sequence persists in more general settings. The optimality of the prices-then-auctions sequence holds even when the sale deadline is stochastic, the seller becomes increasingly impatient, buyers arrive stochastically and have sequential outside options, the mechanism designer is procuring a contract rather than selling, the seller faces separate markets for prices and auctions, or the buyers are long-lived.

This paper contributes to two strands of literature. First, the paper is the first to consider intermingled choices between posted prices and costly auctions. Second, the paper provides a new justification to the use of the buy-it-now selling mechanism. The price-auction sequence has appeared to be optimal in various dynamic settings and the current model is the first to succinctly highlight the importance of the non-stationary endogeneous opportunity cost. Investigations of mechanism choices in the electronic commerce market have received increasing attention in the empirical literature, and the theoretical investigations in this paper can aide future pursuits.

\section{Categories and Subject Descriptors}

J.4 [Social and Behavioral Science]: Economics

\section{General Terms}

Economics

\section{Keywords}

dynamic mechanism design, auction versus price,buy-it-now 